\title{
The Study of Application of Translation Workshop in Translation Teaching of English Majors
}

\author{
Wang Ke*, Wu Jiaping \\ Foreign Language Department, North China Electric Power University, Beijing, China
}

\section{Email address:}

1125865328@qq.com (Wang Ke),jpingwu@126.com (Wu Jiaping)

${ }^{*}$ Corresponding author

\section{To cite this article:}

Wang Ke, Wu Jiaping. The Study of Application of Translation Workshop in Translation Teaching of English Majors. Science Innovation. Vol. 5, No. 5, 2017, pp. 267-271. doi: 10.11648/j.si.20170505.14

Received: June 1, 2017; Accepted: July 5, 2017; Published: July 19, 2017

\begin{abstract}
English teaching must follow the pace of the times. In shortage of translation talents, it is particularly important to improve the translation ability of English majors. However, current translation teaching only emphasizes on the output of translation results, and it still exists many deficiencies. As a new approach to translation teaching, translation workshop pays more attention to the process of translation and improves students' translation level. This method thinks that students are the subject of learning. It focuses on various steps of translation process. The translator should not only learn multi subject knowledge, update their own information, but also develop mutual cooperation and communication skills. The method is helpful to improve the enthusiasm of students' translation, and also conforms to the current direction of translation teaching reform.
\end{abstract}

Keywords: Translation Workshop, English Majors, Translation Teaching

\section{翻译工作坊在大学英语专业翻译教学中的应用研究}

王珂"，吴嘉平

外国语学院, 华北电力大学, 北京, 中国

邮箱

1125865328@qq.com(王珂),jpingwu@126.com(吴嘉平)

摘要：英语教学要紧跟着时代的步伐。在当前翻译人才紧缺的情况下, 提高英语专业学生的翻译水平和翻译能力显得 尤为重要。但当前的翻译教学只重视翻译结果的产出, 存在很多不足之处。“翻译工作坊”作为一种全新的翻译教学方 法, 更加注重翻译的过程, 提高学生的翻译能力和水平。该方法认为学生是学习的主体, 注重翻译过程的各个步骤, 强调译者要学习多学科知识, 及时更新自己的信息, 同时也要培养相互间的合作、交流和沟通能力。这种教学方法不 仅有利于提高学生翻译的积极性, 也符合当前翻译教学改革的方向。

关键词: 翻译工作坊, 英语专业, 翻译教学

\section{1. 引言}

对于中国大学英语专业学生来说, 翻译能力的培养是 十分重要的, 是对英语实践能力的提升。尤其是近年来,
随着国家对外交往程度的加深, 我们与其他国家间的交往 也越来越密切, 政治、经济、科技、文化和贸易等领域的 合作也不断加深。因此对翻译人才的需求也越来越迫切。 而高校外语学院作为国家人才培养的后备军, 需要不断变 革和创新方法, 为国家培养各类翻译人才 $[4]$ 。 


\section{2. 翻译工作坊}

\section{1. 定义}

“工作坊（workshop）”一词最早出现在教育与心理学 的研究领域之中。在19世纪60年代, 美国的劳伦斯 - 哈普 林(Lawence Harplin)将"工作坊"的概念引用到都市计划之 中。一般而言, 工作坊是以一名在某个领域富有经验的主 讲人为核心，10-20名左右的小团体在该主讲人的指导下， 通过活动、讨论、演讲等多种方式, 共同探讨某个话题 [14]。 和传统的教育方式相比, 工作坊有很多好处 [1]。一是探讨 的话题往往更有针对性。很多时候, 都涉及到相关领域的 前沿话题, 甚至, 很多伟大的思想与理论都是在工作坊这 种形式的讨论之中诞生的。二是组织形式更为灵活。可以 在某人的家里, 也可以在正规的会议室里面, 甚至是旅游 景点, 时间与地点都比较灵活。三是费用较为低廉。有时 候，一个工作坊只需参与者支付餐费与场地租用费即可。 至于“翻译工作坊”，根茨勒将其定义为“类似于某种翻译 中心的论坛, 在该论坛上, 两个或两个以上的译者聚集在 一起从事翻译活动。”(Gentzler, 1993:7)。因此, 翻译工作 坊就是一些从事翻译活动的人们聚集在一起, 大家每个人 都有自己的见解和看法, 通过相互讨论和协商, 各抒己见, 确认出比较好的译文文本。这个过程需要大家的沟通和合 作[11]。

我们将翻译工作坊应用到英语翻译教学实践中也是 十分有意义的。传统的翻译教学过于呆板, 有很多不妥之 处。一是只重视翻译结果的输出, 不重视翻译的过程。很 多时候老师直接把翻译的答案给出, 学生不知道为什么这 么翻译, 这样翻译会有什么样的影响等等。缺乏对翻译过 程的体验。二是在传统的翻译课堂中, 老师都是“满堂灌” 或者“一言堂”。学生都是被动地接受知识, 缺乏对学习的 体验: 存疑、选择、批判、探索、想象、和创造。三是忽 视对学生情感的培养。传统的课堂只是注重学习的效果, 对学生的心理环境漠不关心。这种课堂环境比较枯燥、压 抑, 必然会让一些学生失去翻译的兴趣, 感受不到翻译过 程的愉悦。课堂教学脱离学生的生活。单调乏味的理性活 动缺乏应有的生活意义、生命价值和人文关怀。而“翻译 工作坊”式的教学方法可以很好地弥补传统翻译教学方法 的不足。一是“翻译工作坊”属于过程教学法, 注重对翻译 过程的培养。让学生去自主体验和研究翻译的过程, 弄明 白这么翻译的原因。二是老师不再是课堂的主体, 只是课 堂活动的引导者, 学生才是中心。学生们自主讨论译文, 了解自己译文文本的优缺点, 老师在最后对大家不懂的地 方进行讲解。这个过程也培养了学生们的合作能力和沟通 能力, 课堂氛围更加地宽松愉悦。三是有利于培养学生的 创新能力, 激发对翻译的兴趣。学生们分成小组, 每个人 都有自己的任务。在这个过程中, 学生们自主探讨研究, 培养独立思考的能力, 不再像以前那样过于依赖老师。有 两个主要目标: 使学生能够阅读和翻译源语中的文学作 品; 进一步促进智力发展。

由于很多学习外语的人是为了能够看懂外国文学作 品, 所以语法翻译法注重培养学生的阅读和写作能力, 但 其忽视了听说能力的培养。语法翻译法使用母语教学。学
生首先需要学习大量的语法规则, 之后做大量的练习巩固 所学知识, 最后逐字翻译句子。当学生的水平提高时, 他 们就被要求翻译整篇文章。相比翻译内容而言, 语法翻译 法更加注重形式的规范性。在语法翻译法下, 学生们大都 掌握了丰富的语法规则, 基本的语言知识比较扎实, 但实 践和交流能力明显不足。而且只注重形式忽略内容致使学 生们的翻译脱离实际, 显得比较生硬。并且大量记忆各种 复杂的规则给学生带来了很大的负担。长期下去, 学生们 就会失去对翻译的兴趣。

\section{2. 构成}

翻译工作坊由三部分构成: 教师、学生和翻译项目活 动。

\section{（1）教师}

教师既是翻译工作坊的参与者, 又是翻译理论的实践 者。教师在翻译工作坊中起指导作用, 要对学生在翻译过 程中遇到的疑难问题给予及时的指导。

(2) 学生

学生是翻译教学的活动主体。在老师布置完任务之 后, 学生开始自主翻译, 积极参与工作坊的项目活动, 共 同探讨解决翻译文体的方法, 提高团队交流合作能力。

(3) 翻译项目活动

翻译工作坊的项目活动比较广泛, 一般要和学生们未 来的职业发展挂钩。活动形式也比较多, 例如翻译座谈会, 研讨会, 翻译讲座等等[8]。学生们可以通过参加这些活动 增加自己的翻译实践能力, 为以后的职业发展积累经验。 认知法产生于二十世纪六十年代, 由美国著名心理学家约 翰・卡鲁尔提出。卡鲁尔认为学习外语主要基于学生对语 音及语法的分析和学习, 也就是对外语的掌握和控制过 程。在认知法之前, 主流的外语教学方法是听说法。但随 着科技的发展和社会的进步, 单纯机械的听说操练已经不 能满足社会发展的要求。一遍遍的操练会让同学们在保持 兴趣方面难以持之以恒。因此, 听说法逐渐被认知法所取 代。

\section{3. 翻译工作坊在教学中的应用}

在翻译课堂中, 教师要对学生们进行分组, 然后按照 小组把翻译任务分配下去, 各组内同学在翻译完之后对翻 译文本进行讨论, 选取较好的译文, 最后老师进行总结点 评。

\section{1. 课前准备}

在上课前老师要准备好所翻译的材料, 确定教学的重 点难点。翻译材料的选择要尽可能的结合实际, 以应用型 文本为主, 少选取文学性的文本。并且要和学生未来的职 业发展相关, 和翻译市场的需求相结合。在教学重点方面, 教师可以讲解一些翻译理论、技巧和方法, 并提出一些例 子帮助同学们理解。比如, 对于这个句子“to kill two birds with one stone”如果按照异化翻译方法, 可以翻译为“一石 二鸟”，而按照归化的翻译方法，可以翻译成“一箭双雕”, “一举两得”。通过讲解这个例子, 老师可以让同学们理解 
两种翻译方法的不同, 在哪些情况下可以采用归化的翻译 方法, 在哪些情况下可以采用异化的翻译方法。

\section{2. 课堂环节}

在上课过程中, 教师可以首先让各组同学阅读文本, 理解文章的背景, 类型, 风格, 对文章有一个大致的把握。 然后再让大家大声朗读课文, 理解文章大意, 熟悉文章所 要讲解的内容。之后要求学生各自默读课文, 在头脑中进 行大致的翻译, 把不懂的地方划出来, 进行小组讨论[2]。 此外, 遇到不懂的生词, 可以借助字典或其他信息资源, 专业书籍, 翻译软件等, 进行初步翻译。在小组讨论过程 中, 成员分别阅读自己的译文, 并且和大家探讨自己译文 的优缺点, 比如在翻译技巧、方法或者策略方面还有哪些 需要学习和改进的地方, 每组选出一个比较好的译文, 由 小组成员代表进行朗读。其他小组同学可以边听边对比自 己的译文, 看和别人相比有哪些需要改进的地方, 方便大 家学习交流。在这个过程中, 老师是课堂活动的引导者, 给予大家适时的指导。当各组都发言完毕之后, 老师和大 家一起商讨怎样修改译文, 哪些方法用的不合理, 哪些地 方需要改进。如果大家意见一致, 那么就可以对译文进行 修改。如果大家意见不一致, 也可以保留自己的意见, 在 课后进行探讨。

例如，对于这段文章，of course，manufacturing inaccuracies and tooth deflections will cause slight deviations in velocity ratio; but acceptable tooth profiles are based on theoretical curves that meet this criterion. Pitting is initiated when contact stresses are high; it is hastened by sliding action. Its turning radius is small, which means it can work flexibly in narrow space. The combination of high speeds and heavy loads, and for low noise levels. The basic requirement of gear-tooth geometry is the provision of angular velocity ratios. As gears mesh, both rolling and sliding occur, causing pitting and wear. 根据我们上述所说, 首先要让同学们阅 读这段文章, 分析出本文的语体风格。可以看出这是一篇 科技文本。然后大声朗读, 理解本文的大致意思。这篇文 章是有关机械齿轮方面的文章, 讲的是齿轮半径长短和误 差会对机器工作有影响。之后学生们可以默读文章, 大致 进行翻译, 对于不懂的地方要及时的划出来。不懂的生词 也要及时查出来。科技文本一般专业词汇较多, 比如本文 中geometry, velocity, gear等词汇对于一些同学可能难度比 较高, 需要查词典才能知道意思。然后小组成员分别朗读 自己的译文, 大家一起商讨改进的地方, 这也是培养大家 团队协作能力的一种方式 [5]。然后每组派代表发言, 探讨 出大家普遍接受的论文, 老师结合大家的翻译情况进行点 评, 对一些重点难点给予讲解 [10]。

\section{3. 课后修改}

每个同学在课后要提交老师课上点评修改之后的一 份最终译文。这样反复修改可以培养学生的独立思考的能 力, 从而提高自己的翻译水平。老师通过批改同学们的译 文了解同学们对翻译技巧、方法的掌握情况。在这个过程 中, 对于同学们普遍存在的问题老师也要及时进行指导。 对于翻译地比较好的同学要给予鼓励, 提高他们翻译的积
极性[3]。这些不错的译文也可以朗读给全班同学听, 让大 家相互学习。

此外, 在课后也应该注意学生自觉学习能力的培养。 老师也可以要求学生们课下好好复习巩固课上所学新知, 下次进行抽查。学生们也可以通过网络平台相互讨论, 给 老师留言。

\section{4 . 实证研究}

为了研究翻译工作坊在翻译教学中的效果如何, 笔者 对英语专业 50 名本科生进行测试。 25 人为一组, 共两组。 第一组为实验组, 第二组为控制组。在开始前, 先对两个 班学生的翻译能力进行测试。随机抽取课文中的一个小段 落让其翻译, 然后由老师对试卷进行打分。测试结果如下,

表1 实验组和控制组第一次翻译能力测试情况。

\begin{tabular}{lll}
\hline & 实验组 & 控制组 \\
\hline 人数 & 25 & 25 \\
平均数 & 53.02 & 54.85 \\
方差 & 10.01 & 9.96 \\
\hline
\end{tabular}

从以上可以看出, 实验组同学平均成绩为 53.02 , 而 控制组同学平均成绩为 54.85 , 说明控制组学生的翻译总 体水平要高一些。而且从方差也可以看出, 控制组同学的 水平比较稳定。之后, 为了测试翻译工作坊的学习方法是 否有效, 我们对实验组的学生采用翻译工作坊的方式进行 翻译教学, 对控制组的学生采用传统的翻译教学方法。两 组同学的教学内容都是一样的, 时间为八周。之后, 对两 组学生进行测试。每组同学都被要求在 30 分钟内翻译一个 文段。如下, In reviewing our work of the past year, we clearly see that many problems and difficulties remain in our economic and social development. We must always put the interests of the people first. We must truly protect the people's economic, political and cultural rights and interests, pay particular attention to solving acute problems affecting their vital interests, and ensure that poor urban and rural residents have the basic necessities of life. Only if we exercise power for the good of the people can we enjoy the broadest and most reliable support from the people and draw from them the strength for accomplishing all our undertakings. 此段包含 10 个知识点, 分别是语态转换, 倒 装, 增词, 扩展词义, 省略, 否定, 时态, 语序, 语体, 词性转换。测试结果显示, 实验组的同学基本能掌握这十 个知识点。而控制组的同学平均能够掌握五到六个知识 点。下面是两组同学的成绩结果,

表2 实验组和控制组第二次翻译能力测试情况。

\begin{tabular}{lll}
\hline & 实验组 & 控制组 \\
\hline 人数 & 25 & 25 \\
平均数 & 68.23 & 67.07 \\
方差 & 9.93 & 9.99 \\
\hline
\end{tabular}

根据上表的结果, 我们可以看到, 实验组的同学平均 成绩为 68.23 , 而控制组的同学平均成绩为 67.07 . 因此可 以看出, 在这轮的测试中, 实验组学生的平均成绩要明显 高于控制组。在方差的对比中, 实验组的学生是 9.93 , 控 
制组的学生是9.99。说明, 相比控制组的学生, 实验组的 学生结果更加稳定。

因此, 可以看出, 在经过翻译工作坊的教学实践后, 相比控制组的同学, 实验组的同学学习效果更好[12]。在 测试结束后, 对这些不同组的学生分别随机进行采访, 询 问四个问题, 测试他们对教学方法的态度和观点。问题如 下，

1、传统的教学方法和翻译工作坊相比有哪些不同?

学生们认为在传统的教学方法中, 课堂只是老师一个 人在讲, 整个过程不关注学生的回复。学生只是被动的接 受知识, 课堂显得比较乏味。而在翻译工作坊的课堂中, 老师会鼓励学生进行互动, 做一些有意思的任务。学生在 这样的课堂中是主动地接受知识。

2、你认为在翻译教学中运用翻译工作坊可行吗?

所有的受访者都认为在翻译教学中, 采用翻译工作坊 的方法是非常有效的。原因有以下几个方面：1）这种教 学方法有利于同学们取长补短, 尤其是对于基础较差的学 生来说, 可以在和其他同学一起做任务的过程中, 学习别 人的长处, 提高自己的语言运用水平;2）学生们对课堂 上所展示的训练材料非常有兴趣；3）在翻译工作坊的课 堂上, 学生是主体, 因此有很多机会去训练和提升自己的 翻译能力; 4) 翻译工作坊的教学实践是以项目为基础的。 这就和真实的工作环境十分相似。因此在这样的教学方法 下，学生们可以更容易地适应将来的工作环境。

3、在翻译学习中, 你认为翻译工作坊的方法对你翻 译能力的提升有帮助吗?

在采访中, 几乎百分之八十的学生认为翻译工作坊对 提升他们的翻译能力有很大的帮助。他们认为, 翻译工作 坊对提升自己的翻译能力十分有效, 尤其是在翻译过程, 翻译技能和团队合作方面。

4、你能对翻译工作坊的教学方法提出一些建议吗？

受访的学生针对翻译工作坊提出了自己的建议, 主要 有以下几个方面: 1) 教室中的座位方式不适合小组讨论, 建议可以采取圆桌会议的方式让大家面对面的进行交流; 2) 家庭作业是非常有必要的, 因为课堂时间是有限的, 学生们需要有更多的时间去完成翻译任务；3）老师应该 走下课堂, 在合适的时候加入小组讨论, 并给予学生合适 的指导；4）为了激发学生们学习翻译的兴趣, 老师应该 对翻译任务进行仔细的选择。

根据以上测试结果, 我们可以得出, 相比传统的翻译 教学方法 [13], 翻译工作坊的教学效果明显更好。因为老 师给予学生们很大的主动性, 激发学生翻译的灵活性和创 造性, 增加翻译的自主性。就同学们自己而言, 他们也能 接受这种方法, 认为可以很好地提高自己的翻译水平, 培 养自己的团队合作精神。

因此, 我们在之后的翻译教学中, 老师可以考虑把这 种方式应用到教学实践当中以提高学生的翻译水平。

\section{4. 翻译工作坊在教学过程的建议}

(1) 对教师的建议

作为教学过程的引导者, 老师起着至关重要的作用。 翻译教学内容不仅包括对基本翻译理论的学习, 还有对学
生翻译能力和翻译作风的培养。这就要求教师不仅要深谙 各种翻译理论和翻译方法, 还应该将这些理论进行升华, 向学生讲清这样翻译的原因。让学生不仅知其然, 更知其 所以然。

翻译所涉及的不仅仅是语言的转换, 还有多学科知识 的交叉。作为一名教翻译的老师, 不仅要熟悉所转换的语 言, 对各个学科的知识都要有所涉猎, 比如心理学, 社会 学, 认知学, 跨文化交际学, 哲学, 文艺学, 科学等等。 因为翻译文本的类型有很多, 涉及到的知识也十分繁杂。 作为一名老师, 要懂得多学科知识, 这样才可以更好地教 学生。

作为课堂的掌控者, 从事翻译工作坊教学的老师在课 堂上要营造一个自由、民主的氛围, 充分激发同学们的积 极性和创造性[6]。比如, 在小组讨论中, 鼓励同学们积极 地表达自己的想法, 引导他们相互之间进行合作、互动, 取长补短, 互相学习, 这样才会达到良好的学习效果。

作为课堂的创造者, 老师应该帮助学生们创造更多的 学习机会, 激发学生们的学习兴趣和热情。兴趣是最好的 老师, 有了兴趣, 学生们才会更加自主地学习, 提升学习 效果。这就要求老师不仅要熟悉不同的翻译理论还要擅长 翻译实践, 知道如何把不同的理论运用到翻译实践中。

作为课堂的促进者, 老师要帮助学生更好地理解原文 文本, 教导他们如何处理翻译过程中遇到的不同问题, 应 该从哪些方面下手解决等等。这就要求老师具有很高的翻 译能力, 比如要精通源语和目的语, 熟悉翻译技巧等。

作为一名教翻译的老师, 要有在翻译行业工作的经 验。这样才能了解翻译的社会需求和翻译市场的运行情 况。

(2) 对学生的要求

在翻译工作坊的学习中, 学生是课堂活动的主体。所 有翻译项目活动都是围绕学生进行的, 以提高他们的翻译 水平和能力。要想翻译出好的文章, 首先一定要有扎实的 语言功底, 对语言种类要精通, 了解不同国家的语言文化。 比如知道在不同的语境中应该怎么翻译才能够让读者更 加容易接受, 还有一些习语的运用。尤其是中华文化博大 精深, 要想翻译好一些词语或者句子, 必须理解其背后的 文化内涵。比如对于“他是哪吒, 我也要见一见! ”这句话, 出自《红楼梦》, 翻译过来是这样的, “Even if he is a monster, I insist on seeing him.”如果不了解中国文化背景, 很难知道“哪吒”的文化含义, 就没办法翻译。

此外, 学生要培养自己的创新能力。小组活动中要认 真学习, 独立思考, 敢于表达自己的不同想法和见解。在 和大家的相互交流和讨论中迸发出智慧的火花。一名优秀 的译者不仅可以进行语言转换, 而且具有创新思维和创新 能力。否则, 就会在团队合作中受挫, 感受不到自己的价 值所在。一个人只有积极地融入到团队当中, 努力工作, 才会发挥自己的作用。翻译本质上来讲, 也是一种再创造 的过程。所以学生要想成为一名优秀的译员, 必须要培养 自己创造的能力。

学生们除了对语言的学习外, 也要拓宽自己各方面的 知识面。翻译涉及的知识种类很多, 所以要多涉猎各方面 的知识, 完善自己的知识架构。在平时, 要努力学习, 多 多参与课堂活动, 成为课堂活动的主体, 学会与他人合作。 
学生也要多阅读双语书籍提高自己的写作能力。如果 一个译员没有良好的写作水平, 及时了解所翻译的两种语 言, 和不同国家的文化, 是不能准确地把原文翻译出来的。

学生们在翻译中要不断提升自己发现、分析和解决问 题的能力。在翻译工作坊的教学过程中, 每个学生在小组 中都有新的角色, 需要承担自己的责任。所以要充分运用 自己的智慧和创造力克服翻译过程中所面对的各种困难, 翻译出好的作品。

学生要培养自己翻译的激情。如果一个学生热爱翻 译, 他就会花费更多的时间去训练自己, 从而才可以有更 大的进步。相反, 如果没有热情, 只是强迫自己翻译, 那 么效果肯定会大打折扣的。

最后, 学生不仅要学会从自己的翻译实践中总结翻译 的经验, 还要学会在翻译中进行思考。反思自己哪些方面 做的不好, 哪些地方还有改进的地方, 在以后的翻译实践 中就要注意这些地方。学会反思才会促进自己不断进步。

(3) 对翻译任务的要求

翻译任务必须仔细选择, 要符合学生的翻译水平。如 果过难, 就会损伤学生的积极性。如果太过简单, 对于某 些学生不具有挑战性。因此, 选择难度必须适中。

任务选择要贴近生活。这样学生就可以更好地理解原 文文本, 进而翻译输出也就更加准确、恰当、合理。

翻译任务也要和所讲的教学材料相关。比如, 如果本 节课的翻译内容关于文化, 那么翻译任务也应该和文化相 关。

从翻译技巧方面来看, 翻译任务的选择也要谨慎, 这 样才会起到让学生们训练的目的。这就需要老师对学生进 行有针对性地训练。

(4) 对课堂管理的建议

在翻译工作坊课堂中, 学生应该被分成小组, 共同完 成翻译任务。如果老师不知道如何进行课堂管理, 那么课 堂就会显得很混乱。教师在教学过程中要设计和控制每一 步的时间。在翻译工作坊课堂中, 主要包括三个阶段, 教 师要能够预测和控制每一个教学步骤的时间。及时向学生 讲解时间控制和教学步骤, 进而一起协同管理整个过程。

教师要密切关注全班同学, 立即采取有效措施。有时, 一个小组可能大声讨论打扰到邻组, 老师应该提醒他们。 有时一组保持沉默, 教师应该加入, 鼓励学生勇敢地表达 自己的观点。

\section{5. 结论}

翻译是一种实践性很强的活动, 对译者的要求很高。 翻译工作坊作为一种新式的教学手段, 可以很好的应用到 高校翻译课堂中[7]。从我们的案例研究中, 也可以看出,
这种方式让学生成为课堂的主体, 锻炼学生独立思考和学 习的能力, 减少对老师的依赖, 激发他们对翻译的热情, 有效地提高学生的翻译水平。尤其是在当前翻译人才紧缺 的情况下, 可以培养出优秀的翻译人才 [9], 创造良好的教 学效果。

\section{参考文献}

[1] 翻译工作坊教学探微J] 李明, 仲伟合. 中国翻译. 2010 (04) : (32-36+95)。

[2] “翻译作坊”在翻译教学中的运用 $[\mathrm{J}]$. 肖红. 四川外语学院 学报. 2005 (01)：(139-142)。

[3] 浅谈“翻译工作坊式”翻译实践教学模式 $[J]$. 宋平峰. 内蒙 古农业大学学报(社会科学版). 2011 (01): (140-141+143)

[4] 大学英语翻译教学现状分析及改革思考 [J]. 王玉玨, 胡 江．科教文汇(上旬刊). 2010（08）:(105-106)

[5] 以翻译工作坊模式促进翻译课堂教学[J]. 陈宣荣. 教育教 学论坛. 2012 (S4)：(170-171)。

[6] 翻译工作坊核心目标及其实现路径尝试 [J]. 范东生. 合 肥师范学院学报. 2014（04）：（77-81）。

[7] 翻译工作坊在翻译教学中的实践模式探讨 [J]. 白美姻. 吉 林省教育学院学报. 2017（02）：（76-79）。

[8] 关于当前中国翻译教学研究的思考 [J]. 薄振杰, 李和庆. 外 语界. 2011（05）：(76-83）。

[9] 翻译专业人才培养模式探索与实践 [J]. 仲伟合, 穆雷. 中 国外语. 2008（06）：(4-8+14）。

[10] 从翻译课堂到翻译工作坊——翻译精品课程建设的启示 [J]. 张春柏, 吴波. 外语教学理论与实践. 2011（02）: (70-73)。

[11] 翻译工作坊教学模式的构建与实践 [J]. 曾丽馨, 孙晓芸. 安 徽工业大学学报（社会科学版）.2013(02)。

[12] 云计算学习平台下MTI翻译工作坊教学模式研究 [J]. 董洪 学, 张坤媛. 外语电化教学. 2016 (01): (56-61)。

[13] 翻译工作坊模式与应用型翻译人才培养 [J]. 刘瑞, 杨楠. 黑 龙江教育学院学报． 2016(09):（115-117）。

[14] “翻译工作坊”在翻译硕士专业(MTI)教学中的应用研究 [J]. 郭淑丽, 贾存, 爱张倩. 科技风. 2017(03): (296-297)。 